\title{
Progressive introgression between Brassica napus (oilseed rape) and $B$. rapa
}

\author{
LB Hansen ${ }^{1}$, HR Siegismund ${ }^{2}$ and RB Jørgensen ${ }^{3}$ \\ ${ }^{1}$ Botanical Institute, Øster Farimagsgade 2D, DK-1353 Copenhagen K, Denmark; ${ }^{2}$ Department of Evolutionary Biology, University of \\ Copenhagen, Zoological Institute, Universitetsparken 15, DK-2100 Copenhagen Ø, Denmark; ${ }^{3}$ Risoe National Laboratory, Plant \\ Research Department, Frederiksborgvej 399, PO Box 49, DK-4000 Roskilde, Denmark
}

\begin{abstract}
We have earlier shown extensive introgression between oilseed rape (Brassica napus) and $B$. rapa in a weedy population using AFLP markers specific for the nuclear genomes. In order to describe the progress of this introgression, we examined 117 offspring from 12 maternal plants from the introgressed population with the same AFLPmarkers; AFLP data were supported by chromosome counting. We also analysed the offspring with a speciesspecific chloroplast marker and finally evaluated the reproductive system in selected maternal plants. Our results indicated a high outcrossing rate of the introgressed maternal plants. It seemed that $B$. rapa most often functioned as the
\end{abstract}

maternal plant in the introgression process and that the amount of oilseed rape DNA was highly diminished in the offspring compared to their introgressed maternal plants. However, our analysis of plants from the weedy population indicated that introgression can lead to both (1) exchange of chloroplast DNA between species producing $B$. rapa-like plants with $B$. napus chloroplasts and (2) incorporation of $B$. napus $C$-genome DNA into the $B$. rapa genome. Therefore, we question whether it can be regarded as containment to position transgenes in the chloroplast or in specific parts of the nuclear genome of $B$. napus.

Heredity (2003) 91, 276-283. doi:10.1038/sj.hdy.6800335

Keywords: AFLP; reproductive system; risk assessment; cpDNA; chloroplast capture; hybridisation

\section{Introduction}

Genetically modified varieties of the important crop oilseed rape (Brassica napus) are on the variety list in several countries (eg USA and Canada). Oilseed rape has many closely related weedy species, and at least two of these, $B$. rapa and Raphanus raphanistrum hybridise spontaneously with oilseed rape (Jørgensen and Andersen, 1994; Chévre et al, 1998). This has raised concerns about the risk of spread of transgenes from oilseed rape to weedy relatives, which in some cases could increase their weediness.

B. napus is an allotetraploid species $(2 n=38$, genome constitution AACC), which has probably evolved through hybridisation and polyploidisation between the two diploid species $B$. rapa $(2 n=20$, AA) and $B$. oleracea $(2 n=18, C C)(U, 1935)$. The difference in ploidy level between $B$. napus and $B$. rapa is expected to have an impact on the introgression process through the production of unbalanced gametes. A comparison with the ancestral genomes revealed that the B. napus chromosomes have been rearranged (Cheung et al, 1997), and it has been hypothesised that the rearrangements occurred immediately after polyploidisation of the $B$. rapa $\times B$. oleracea hybrid (Lagercrantz, 1998). If such rearrangements also occur early in the introgression between

Correspondence: RB Jørgensen, Risoe National Laboratory, Plant Research Department, Frederiksborgvej 399, PO Box 49, DK-4000 Roskilde, Denmark. E-mail: rikke.bagger.jorgensen@risoe.dk

Received 10 May 2003; accepted 15 April 2003 ploidy levels, it is likely that introgression will lead to recombination between the $B$. napus and B. rapa genomes. Therefore, the position of transgenes on $C$ chromosomes may not be the way to exclude spread of transgenes from oilseed rape, because this presupposes that there is no recombination between the $\mathrm{A}$ and $\mathrm{C}$ genomes in oilseed rape or in the interspecific hybrids. Placing the transgene in the chloroplast instead of the nuclear DNA could be an alternative method to reduce gene flow (Daniell et al, 1998), since in Brassica, like most other angiosperms, chloroplast DNA is not transmitted by the pollen (Corriveau and Coleman, 1988). Hypothetically, plastidencoded transgenes could be transferred from $B$. napus to $B$. rapa, if $B$. napus was the female just once in the introgression process (Scott and Wilkinson, 1999). In fact, only two generations of crossing $\left(\mathrm{F}_{1}, \mathrm{BC}_{1}\right)$ with $B$. napus acting as the initial maternal plant could produce B. rapa-like plants, which carry 20 A chromosomes and no C chromosomes (Mikkelsen et al, 1996) and the B. napus chloroplast DNA. Our present findings are the first to show such chloroplast capture in nature.

In a previous study we found pronounced introgression in a mixed population of $B$. napus and $B$. rapa (Hansen et al, 2001). Our results suggested that the introgression had been in progress for several years. Plants from this mixed population were used in the present study to examine how introgression progressed between two generations. Furthermore, the reproductive system in the introgressed plants was studied. 


\section{Materials and methods}

\section{Plant material}

Offspring from 12 maternal plants were grown from naturally pollinated seeds harvested in a mixed weedy population of $B$. napus and B. rapa. It was located in a 4 ha organic field at Sjællands Odde in the Northwestern part of Zealand, Denmark. This field had been cultivated organically since 1987 and we collected the material in 1998, where a mix of pea, clover and barley was cultivated. Different species grew as weeds in the field and were distributed in a patchy pattern with the mixed population of $B$. napus and $B$. rapa covering about $20 \%$ of the field. The densities of weedy B. napus and B. rapa were high, about $20-40$ plants $/ \mathrm{m}^{2}$. In total, more than 160000 weedy Brassica plants were present at the site. Oilseed rape has not been cultivated at the field since it was converted to organic cultivation; therefore, the current weedy $B$. napus are most likely the remnants from when the field was cultivated conventionally. In 1995, RAPD data generated from this population suggested introgression (Landbo, 1997).

We have previously investigated 102 plants from this population for introgression with species-specific AFLPmarkers (Hansen et al, 2001). These plants were collected in the centre of the population, where they represented all Brassica-like plants within $3 \mathrm{~m}^{2}$. The AFLP-profiles of the plants showed that seven were $B$. napus-like, while 50 were B. rapa-like, having only B. napus or B. rapa-specific markers, respectively. Moreover, 45 were introgressed having both $B$. napus- and $B$. rapa-specific markers (Hansen et al, 2001). One of the introgressed plants was a first-generation $\left(\mathrm{F}_{1}\right)$ hybrid, whereas all the remaining had introgressed beyond the $\mathrm{F}_{1}$ stage.

The 12 maternal plants of the offspring for the present study were: four B. napus (\#9, 13, 20, 42), seven introgressed plants (\#31, 38, 45 (the $F_{1}$ hybrid), 66, 76, $83,101)$ and one $B$. rapa (\#87). The maternal plants were selected to represent both introgressed and apparently pure species, with the majority being introgressed as the focus was on the progression of the introgression process. The introgressed plants had as distinct AFLP markers and physical distributions within the collection area as possible. The oilseed rape plants were also scattered at the site. As oilseed rape was infrequent, it was probably exposed to heavy loads of $B$. rapa or introgressed pollen. Therefore, we chose to analyse more offspring families from B. napus compared to B. rapa. In all, 10 offspring from each maternal plant, sired in the field, were screened with AFLP markers, except \#83 where seven offspring were screened. In addition, all the 102 maternal plants previously studied were screened using a species-specific chloroplast DNA (cpDNA) marker. For those maternal plants showing a chloroplast type conflicting with their AFLP profile, offspring were also screened with the cpDNA marker. Chromosomes were counted in 18 offspring from five of the introgressed maternal plants (maternal plants: \#38, 45, 66, 76, 83). These offspring plants were selected so that three had no $B$. napus-specific AFLP markers (results not shown), whereas the remaining 15 had both $B$. napus- and $B$. rapa-specific markers.
Seed germination

The seeds were germinated in Petri dishes on moist filter paper. Seed dormancy was broken by temperature cycling in a $0.2 \% \mathrm{KNO}_{3}$ solution: $8 \mathrm{~h}$ at $30^{\circ} \mathrm{C}$ followed by $16 \mathrm{~h}$ at $20^{\circ} \mathrm{C}$. Seeds were kept in darkness during germination. The seedlings were pricked out into soil, whereupon they were grown in a greenhouse with $8 \mathrm{~h}$ darkness at $15^{\circ} \mathrm{C}$ and $16 \mathrm{~h}$ light at $20^{\circ} \mathrm{C}$.

\section{Chromosome counting}

Chromosomes were counted from meiotic (pollen mother cells) and mitotic (root meristematic cells) cell divisions. Flower buds were picked when they had reached one quarter of mature size and preparations for chromosome counting at metaphase I were conducted, as described by Linde-Laursen and Bothmer (1997). Analysis of somatic chromosomes was made on root tips pretreated in a $0.05 \%$ colchicine solution for $2 \mathrm{~h}$. The root tips were stained with Feulgen and squashed in $45 \%$ acetic acid.

\section{DNA extraction, AFLP procedure and PCR analysis of the chloroplast markers}

The DNA extraction was made from fresh or frozen leaves using the procedure described by Edwards et al (1991), with the modifications described by Johannessen et al (2002). The AFLP procedure was conducted according to Vos et al (1995), with the modifications also described by Johannessen et al (2002). The preamplifications were carried out with one selective nucleotide and in the specific amplification, two or three nucleotides were added to the primer. Adaptors were as described by Maughan et al (1996). Primer combinations tested and development of the species-specific AFLP markers are described in detail in Hansen et al (2001). Four primer combinations (I-IV) were used, which collectively resulted in approximately 500 AFLP bands and provided 24 species-specific nuclear markers: three markers specific for the B. rapa A genome [two polymorphic (I1r, II-1r) and one monomorphic (IV-1r)] and 21 B. napusspecific markers. In total, 18 of the $B$. napus-specific markers were specific for the $\mathrm{C}$ genome (C-markers) and three were specific for $B$. napus per se (n-markers); it is not known whether these three markers are located on the $A$ or $\mathrm{C}$ genomes. Most of the oilseed rape markers were monomorphic (Hansen et al, 2001; Tables 1 and 2). For investigation of the reproductive system no special markers were developed but the total set of AFLP bands were used. For each offspring, it was established whether it was descended from selfing by comparing the total AFLP-banding pattern $(\sim 500$ from the four primer combinations) to the AFLP-banding pattern of the maternal plant. An offspring was regarded as descended from outcrossing when it had five or more bands not present in the maternal plant. The outcrossing rate was measured in each family as the number of offspring descending from outcrossing relative to the total number of offspring from the maternal plant.

For analysis of plastid origin, the chloroplast markers and the PCR procedure described in Taberlet et al (1991) were applied. The marker we used was bounded by primers ' $\mathrm{e}$ ' and ' $\mathrm{f}$ ' as they provided species-specific markers for $B$. napus and $B$. rapa. The specificity of the chloroplast marker was tested on the same nine popula- 
tions of $B$. rapa and nine varieties of oilseed rape that were used to develop the AFLP markers (Hansen et al, 2001). For some plants we also verified the origin of the cytoplasm by applying an AFLP marker considered to be specific for the B. rapa cytoplasm. This marker (III-4r, EAC/M-CAG) was found by chance when the AFLP markers were developed, and verified as being specific to the cytoplasm by screening nine different hybrid families between oilseed rape and $B$. rapa with one or the other species as the mother.

\section{Results}

\section{Inheritance of species-specific AFLP markers}

The 10 offspring from the maternal B. rapa plant were all pure $B$. rapa according to the species-specific markers. Out of 67 offspring, from introgressed plants, 24 offspring had inherited both $B$. napus and $B$. rapa-specific markers (Table 1), whereas the remaining 43 were $B$. rapa-like having inherited only $B$. rapa-specific markers (not included in Table 1). Among the 40 offspring from $B$. napus maternal plants there were six that, in addition to the B. napus-specific markers, also carried B. rapa-specific markers (Table 2). Thus, from a total of 117 offspring screened, 30 had both B. napus- and B. rapa-specific markers, 34 had only $B$. napus-specific markers and the remaining 53 only $B$. rapa-specific markers. Offspring that showed B. napus-specific markers that were not found in their introgressed maternal plant, must have descended from pollination by introgressed pollen. For the remaining group, consisting of introgressed offspring with no species-specific markers different from the maternal plant, we cannot assess whether they descended from fertilisation by introgressed pollen or B. rapa pollen (Tables 1 and 3). Generally, the offspring had fewer B. napus-specific markers than their maternal plants. Table 3 summarises the identity of the male parent of the offspring, deduced from the combined information from the species-specific markers with most of the markers being monomorphic (Tables 1 and 2).

\section{Chromosome counting}

Between four and 11 mitotic cell divisions were counted in each of the 15 offspring that had both B. napus- and $B$. rapa-specific markers (Table 1 ). In addition, meiotic cells were counted from two of the offspring (\#45-24, nine meiotic cells; \#45-25, three meiotic cells) that had 26 and 25 chromosomes in the mitotic cell divisions, respectively. For both plants, all the meiotic cells had nine or 10 bivalents, most likely representing A chromosomepairing, and between five and seven univalents, probably representing C chromosomes. Moreover, chromosomes were counted in three offspring that had only $B$. rapa-specific markers but descended from introgressed maternal plants; two of these had 20 chromosomes, whereas the third (\#66-10) had 21.

\section{Outcrossing rate}

All offspring of introgressed plants had between two and 13 AFLP bands that were not present in the maternal plant, and 24 of the 40 offspring from B. napus had between one and 10. All offspring from B. rapa had five to 13 extra AFLP bands. From these results we assess all the
B. rapa offspring to descend from outcrossing. However, since the AFLP technique could produce aberrant bands, offspring plants from $B$. napus and introgressed plants were not considered as being outcrossed if they had fewer than five nonmaternal bands. The threshold of five bands was chosen because this was the lowest number of non-maternal bands found in offspring from the selfincompatible B. rapa. This produces estimated outcrossing rates of 40 and $80 \%$ for B. napus and introgressed offspring, respectively.

\section{Chloroplast specificity}

As is known from the oilseed rape breeders (ie Andreas Gertz, DLF-Trifolium, St Heddinge), some of the oilseed rape varieties are bred to harbour a $B$. rapa cytoplasm. Using the cpDNA marker this was confirmed for two of the varieties (Optima and Line) in the test array. All other varieties had the oilseed rape cytoplasm. Eight of the populations in the test array of $B$. rapa had the expected cytoplasm but in the ninth population (Bc 29, a large weedy population in an oilseed rape field, Figure 1), nine out of 23 individuals revealed a B. napus cytoplasm. These nine individuals had neither AFLP markers specific to B. napus (Hansen et al, 2001) nor the AFLP marker specific to the B. rapa cytoplasm (III4r, E-AC/MCAG).

Of the 102 plants from the natural population, 91 were cpDNA-typed. In Table 4, the results are shown together with the species assessment (introgressed, B. napus or $B$. rapa) from a previous AFLP analysis of the same plants (Hansen et al, 2001). Among the 91 plants from the natural population all seven plants assessed to be $B$. napus from the AFLP profile had $B$. napus-chloroplasts, whereas one of the 47 plants assessed to be B. rapa (\#35) unexpectedly had B. napus chloroplasts. Regarding the plants that were assessed to be introgressed based on their AFLP profiles, all but two of 36 plants carried $B$. rapa chloroplasts. One of these two individuals was the $\mathrm{F}_{1}$ hybrid (\#45), whereas the other (\#54) was introgressed beyond the $F_{1}$ stage. As plant \#54 did not produce any fertile seeds, analysis of offspring was not possible. A total of 10 offspring from each of plants \#35 and \#45 were screened and all their offspring carried, just as the maternal plant, B. napus cpDNA (Figure 2).

\section{Discussion}

In an introgressed population, like the one studied here, it is interesting to know the breeding system of the introgressed plants since pronounced outcrossing would lead to a higher level of gene exchange between the two species than if the plants were primarily selfing (Levin, 1978). From the AFLP profiles it was estimated that introgressed plants had an outcrossing rate of at least $80 \%$. Concerning B. rapa and B. napus, outcrossing rates were as expected, with $B$. rapa having sporophytic selfincompatibility (Bateman, 1955) and B. napus selfing to a large extent (Olsson, 1952). To avoid overestimating the outcrossing rates, the possibility that AFLPs might produce erroneous bands has been considered, and only offspring with at least five nonmaternal bands were accepted as being from outcrossing. However, outcrossing could also be underestimated, as the AFLP technique produces dominant markers. 
Table 1 Introgressed offspring from introgressed maternal plants (\#=maternal plant)

\begin{tabular}{|c|c|c|c|c|c|c|c|c|c|c|c|c|c|c|c|c|c|c|c|c|c|c|c|c|c|c|c|c|c|}
\hline Marker & $\# 45$ & $-3-4$ & $-4-6$ & $6-7$ & -20 & -21 & -22 & -23 & -24 & -25 & $\# 66$ & $-5^{a}$ & -6 & $-9^{n} \quad$ & \#101 & $-9^{a}-$ & $-10^{a}$ & $\# 31$ & $-5^{a} \#$ & $\# 76$ & $-6^{a}$ & $-7 \# 3$ & -21 & $\# 83$ & -3 & & -5 & -6 & -7 \\
\hline $\mathrm{I}-1 \mathrm{r}(\mathrm{p})$ & $x$ & $x$ & & $x$ & $x$ & $(X)$ & $(X)$ & $(\mathrm{X})$ & $(X)$ & $(\mathrm{X})$ & $x$ & & $x$ & $x$ & $x$ & $x$ & $x$ & $x$ & & & & $x$ & $x$ & $x$ & $x$ & $x$ & $x$ & $x$ & $x$ \\
\hline II- $1 \mathrm{r}(\mathrm{p})$ & & $x$ & $x$ & & & $x$ & & $x$ & $x$ & $x$ & $x$ & $x$ & $x$ & $x$ & $x$ & $x$ & $x$ & $x$ & $x$ & $x$ & $x$ & $x \times$ & $x$ & $x$ & $x$ & $x$ & $x$ & $x$ & $x$ \\
\hline IV-1r & $x$ & $x \times$ & $x \quad x$ & $x$ & $x$ & $x$ & $x$ & $x$ & $x$ & $x$ & $x$ & $x$ & & $x$ & $x$ & $x$ & $x$ & $x$ & $x$ & $x$ & X & $x \times$ & $x$ & $x$ & $x$ & $x$ & $x$ & $x$ & $x$ \\
\hline II- $2 n$ & $x$ & $X \times$ & $X \quad X$ & & $\mathrm{X}$ & & $x$ & $x$ & $\mathrm{X}$ & $x$ & & & & $x$ & & & $x$ & & & & & $x$ & & $x$ & & & & & \\
\hline II- $3 \mathrm{n}$ & $x$ & $x \times$ & $x \quad x$ & & $x$ & & & & & & & & & & $x$ & & $x$ & & & & & & & $x$ & & & & & \\
\hline III-1n & $x$ & $x \times$ & $x \quad x$ & $x$ & & $x$ & $x$ & $x$ & $x$ & $x$ & & & & & & & & & & & & & & & & & & & \\
\hline $\mathrm{I}-1 \mathrm{C}$ & $x$ & $x$ & $x$ & & & $x$ & & $x$ & & $x$ & & & & & $x$ & & & & & & & & & & & & & & \\
\hline $\mathrm{I}-2 \mathrm{C}(\mathrm{p})$ & $x$ & $x$ & $x$ & & $x$ & $x$ & $x$ & & $x$ & $x$ & & & & & & & $x$ & & & $x$ & & $x$ & & & & & & & \\
\hline I- $4 \mathrm{C}$ & $x$ & $x \times$ & $x \times$ & & & $x$ & $x$ & $x$ & $x$ & $x$ & & & & & $x$ & & & & & & & & & & & & & & \\
\hline $\mathrm{I}-6 \mathrm{C}$ & $\hat{x}$ & $x \times$ & $x$ & & $x$ & & $x$ & $x$ & $\hat{x}$ & $x$ & & $x$ & & $x$ & & & $x$ & & & & $x$ & & & $x$ & & & & & $(\mathrm{X})$ \\
\hline II-2C & $x$ & & & $x$ & & & (X) & $x$ & & & & & & & $x$ & & & & & & & & & & & & & & \\
\hline II-3C & $x$ & $x \times$ & $x \quad x$ & & $x$ & $x$ & $x$ & $x$ & $x$ & $x$ & $x$ & & $x$ & X & $x$ & & & $x$ & & $x$ & & $x$ & & $x$ & & & & & \\
\hline II-6C & $\hat{x}$ & $x \times$ & $x \times$ & & $\hat{x}$ & & $x$ & $x$ & $x$ & $x$ & & & & & & & & & & & & & & & & & & & \\
\hline II-7C & $x$ & $x \times$ & $x \quad x$ & $(X)$ & $x$ & $x$ & & $x$ & $\mathrm{X}$ & $x$ & & & & & & & & & & & & & & & & & & & \\
\hline II-10C (p) & & & & & & & & & & & & & & & & & & & & & & & & $\mathrm{x}$ & & & & & \\
\hline III-2C & $x$ & $x \times$ & $X \times$ & & $x$ & $x$ & $X$ & $x$ & 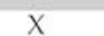 & $x$ & & & & & & & & & & & & & & & & & & & \\
\hline III-3C & $x$ & $x \times$ & $x \quad x$ & $x$ & $x$ & $x$ & $x$ & $x$ & $x$ & $x$ & & & & & & & & & & & & & & & & & & & \\
\hline IV-1C & $x$ & $x \times$ & $x \times$ & & $x$ & & $x$ & $x$ & $x$ & $x$ & & & & & & & & & & $x$ & & & & & & & & & \\
\hline IV-2C & $x$ & $x$ & $x$ & & & & $x$ & $x$ & & & & & & & $x$ & & & & & & & & & $x$ & $x$ & $x$ & $x$ & $x$ & $x$ \\
\hline IV-3C & $x$ & $x$ & $x$ & & $x$ & & $x$ & $x$ & & & & & & & & & & & & $x$ & & $x$ & & & & & & & \\
\hline IV-4C & $x$ & $x \times$ & $x \quad x$ & & & & $x$ & $x$ & $x$ & $x$ & & & & $x$ & & & & & & & & & & & & & & & \\
\hline IV-5C & $x$ & & $x$ & & $x$ & & $x$ & & & & & & & & & $x$ & & & $\times \gamma$ & $x$ & & $x \times$ & & & & & & & \\
\hline IV-6C & $x$ & $x$ & $x$ & & $x$ & & $x$ & & $x$ & $x$ & $x$ & & & $x$ & & & & & & $x$ & & $x$ & $x$ & & & & & & \\
\hline IV-7C (p) & $x$ & & & & & & $x$ & $x$ & & & & & & & & & & & & $x$ & & & & & & & & & \\
\hline $2 n=$ & & & & & 25 & 25 & 26 & 26 & 26 & 25 & & 20 & 22 & 21 & & & & & & & 20 & & 23 & & 20 & & 21 & 21 & 21 \\
\hline variation & & & & & $22-26$ & $23-26$ & $25-26$ & $24-30$ & $25-26$ & 25 & & $20-22$ & $20-22$ & $20-22$ & & & & & & & $20-21$ & & $22-24$ & & $20-21$ & & $20-22$ & $20-21$ & $20-22$ \\
\hline
\end{tabular}

affspring descended from pollination by introgressed pollen, as they have B. napus-specific markers not present in the maternal plants. \#45, an $\mathrm{F}_{1}$ hybrid. The line-hatched cells indicate markers that could not be read due to blurred gels and brackets designates uncertainty of reading. Markers followed by (p) are polymorphic, whereas the remaining are monomorphic.h: $r$. B. napus specific markers; $n$ and C. B. napus specific markers. 
Table 2 Introgressed offspring from B. napus maternal plants. (\#=maternal plant)

\begin{tabular}{|c|c|c|c|c|c|c|c|c|c|}
\hline Markers & $\# 13$ & -1 & \#20 & -6 & -8 & -10 & -13 & $\# 42$ & -10 \\
\hline $\mathrm{I}-1 \mathrm{r}(\mathrm{p})$ & & & & & $x$ & & & & \\
\hline II- $1 \mathrm{r}(\mathrm{p})$ & & $x$ & & $x$ & $\mathrm{X}$ & $x$ & & & \\
\hline IV-1r & & $x$ & & $x$ & $x$ & $X$ & $X$ & & $x$ \\
\hline II- $2 n$ & $x$ & $x$ & $x$ & $x$ & $x$ & $x$ & $x$ & $x$ & $x$ \\
\hline II-3n & $x$ & $x$ & $x$ & & $x$ & $x$ & & $x$ & $x$ \\
\hline III-1n & $x$ & $x$ & $x$ & $x$ & $x$ & $x$ & $x$ & $x$ & $x$ \\
\hline I-1C & $x$ & $x$ & $x$ & $x$ & $x$ & $x$ & $x$ & $x$ & $x$ \\
\hline $\mathrm{I}-2 \mathrm{C}(\mathrm{p})$ & $x$ & $x$ & $x$ & $x$ & & $x$ & $x$ & $x$ & $x$ \\
\hline$\left[-4 C^{2}\right.$ & $x$ & $x$ & $x$ & $x$ & $x$ & $x$ & $x$ & $x$ & $x$ \\
\hline I- $6 \mathrm{C}$ & $x$ & $x$ & $x$ & $x$ & $x$ & $x$ & $x$ & $x$ & $\mathrm{x}$ \\
\hline II-2C & $x$ & $x$ & $x$ & & $x$ & $x$ & & $x$ & $x$ \\
\hline II-3C & $x$ & $x$ & $x$ & $x$ & $x$ & $x$ & $x$ & $x$ & $x$ \\
\hline II-6C & $x$ & $x$ & $x$ & $x$ & $x$ & $x$ & $x$ & $x$ & X \\
\hline II-7C & $x$ & $x$ & $x$ & $x$ & $x$ & $\mathrm{x}$ & $x$ & $x$ & $x$ \\
\hline II-10C (p) & $x$ & & & & & & & $x$ & $x$ \\
\hline III-2C & $x$ & $x$ & $x$ & $x$ & $x$ & $x$ & $x$ & $x$ & $x$ \\
\hline III-3C & $x$ & $\mathrm{x}$ & $x$ & $x$ & $x$ & $x$ & $x$ & $x$ & $x$ \\
\hline IV-1C & $x$ & $x$ & $x$ & $x$ & $x$ & $x$ & $x$ & $x$ & $x$ \\
\hline IV-2C & $x$ & $x$ & $x$ & $x$ & $x$ & $x$ & $x$ & $x$ & $x$ \\
\hline IV-3C & $x$ & $\mathrm{X}$ & $x$ & $x$ & $x$ & $x$ & $x$ & $x$ & $\mathrm{x}$ \\
\hline IV-4C & $x$ & $x$ & $x$ & $x$ & $x$ & $x$ & $x$ & $x$ & $x$ \\
\hline IV-5C & $x$ & $\mathrm{x}$ & $x$ & $x$ & $x$ & $x$ & $x$ & $x$ & $\mathrm{X}$ \\
\hline IV-6C & $x$ & $\mathrm{X}$ & $x$ & $x$ & $x$ & $x$ & $x$ & $x$ & $x$ \\
\hline IV-7C (p) & & & $x$ & & $x$ & & & & \\
\hline
\end{tabular}

\#20-6 and \#20-13 did not show the monomorphic markers II-3n and II-2C, which indicated that this marker was not monomorphic in the weedy population or that \# 20 was an introgressed heterozygote. See footnote of Table 1.

Table 3 Paternity and number of introgressed offspring

\begin{tabular}{|c|c|c|c|c|}
\hline $\begin{array}{l}\text { Plant } \\
\text { identity }\end{array}$ & $\begin{array}{l}\% \text { in maternal } \\
\text { population }\end{array}$ & $\begin{array}{l}\text { Maternal plants/offspring/ } \\
\text { introgressed offspring }\end{array}$ & $\begin{array}{l}\text { Male parent } \\
\text { of offspring }\end{array}$ & $\begin{array}{l}\text { Outcrossing } \\
\text { rate } \%\end{array}$ \\
\hline $\mathrm{F}_{1}$ hybrid & 1 & $1 / 10 / 10$ & All int. or B.r & 100 \\
\hline Introgressed beyond $F_{1}$ stage & 43 & $6 / 57 / 14$ & 14 int. or B.r/43 B.r. & $\geqslant 80$ \\
\hline B. rapa & 49 & $1 / 10 / 0$ & All B.r. & 100 \\
\hline B. napus & 7 & $4 / 40 / 6$ & 6 int. or B.r/34 B.n. & $\geqslant 40$ \\
\hline
\end{tabular}

B.r. Brassica rapa; B.n. B. napus; int. . introgressed plants.

The high outcrossing rate in the introgressed plants could be due to either inheritance of the sporophytic self-incompatibility system functioning in $B$. rapa or to dysfunction of self-pollen caused by unbalanced chromosome number in the gametes. Whatever the reason, the high outcrossing rate could favour the spread of introgressed gametes into the population.

Among the offspring from the introgressed maternal plants only one-third had both $B$. napus- and B. rapaspecific markers (Table 1). Of the introgressed offspring, only six were with certainty assessed to descend from pollination by introgressed pollen (Table 1). This suggests that $B$. rapa was the most common father. Alternatively, some of the offspring that were apparently derived from pollination by B. rapa may have descended from siring by introgressed plants, in which $B$. napus DNA has been eliminated in the gametogenesis. Among the 67 ovules from introgressed plants, $43 \mathrm{~B}$. rapa-like and four introgressed ovules (see Table 1) lacked all the maternal (and monomorphic) B. napus markers; this possibly resembles the elimination of $B$. napus DNA in pollen.
The chromosome counting revealed that all the chromosomes in the $B$. napus-genome had not been covered with AFLP markers. For example, offspring \#3821 had 23 chromosomes, probably 20 A chromosomes and three $\mathrm{C}$ chromosomes, but only one $B$. napus-specific marker. Offspring \#66-9 had five B. napus-specific markers but only one chromosome in addition to the 20 A chromosomes expected. These five markers could be located on the same chromosome or they could have been recombined into the $B$. rapa genome. Three offspring (\#66-5, \#76-6, \#83-3; Table 1) that each had one $B$. napus-specific marker located on the $C$ genome ( $C$ marker) had only 20 chromosomes. This suggests that the $B$. napus $\mathrm{C}$ genome was recombined into the $B$. rapa $\mathrm{A}$ genome.

B. napus DNA was eliminated to a high degree in gametogenesis in the introgressed plants, leaving only a few gametes with $B$. napus DNA. However, many offspring typed as $B$. rapa could be introgressed, since the $B$. napus markers do not cover the crop genome and introgression would go undetected. In this case, the introgression frequency would be higher than the results suggest. 


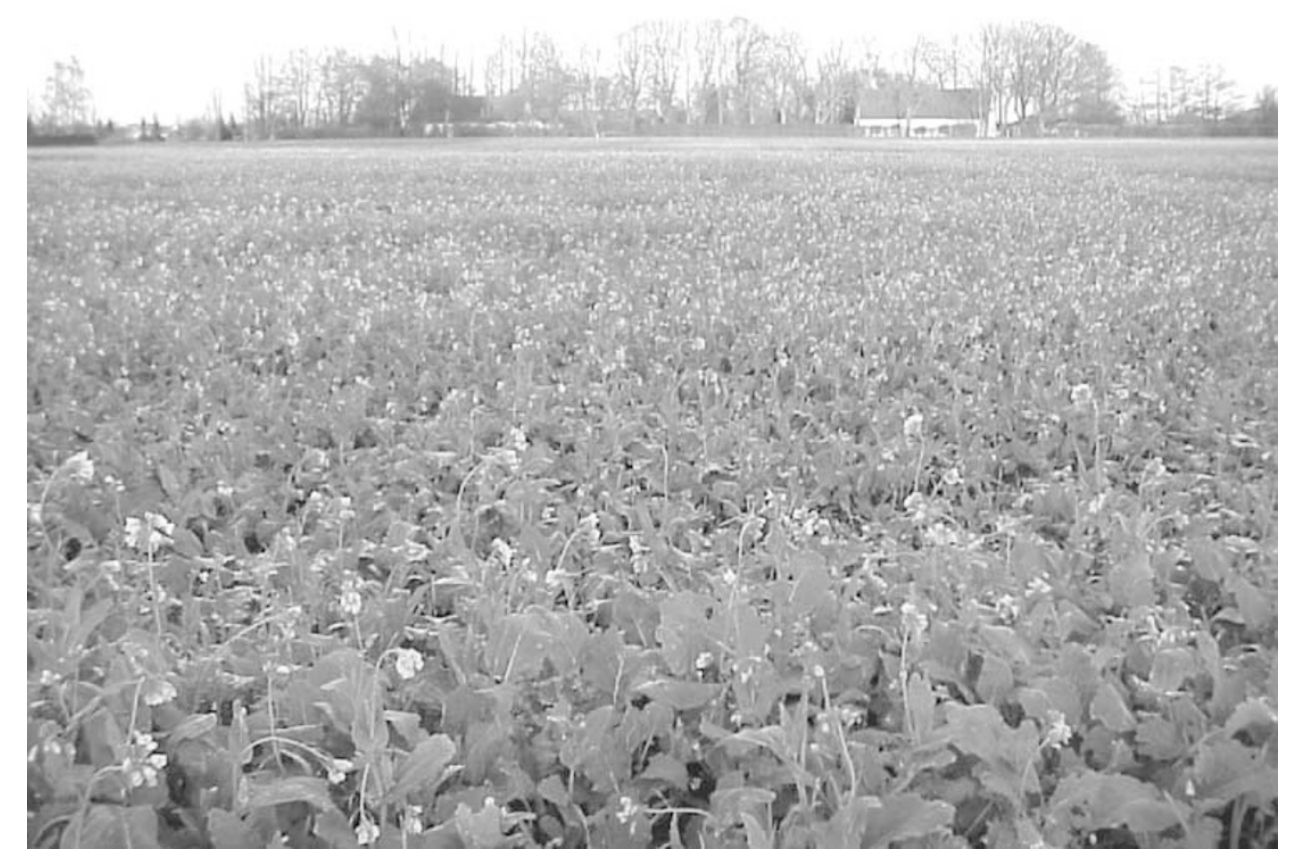

Figure 1 Autumn-flowering weedy B. rapa (population Bc 29) in a winter oilseed rape field in October. Populations of $B$. rapa may flower in the spring, together with oilseed rape and then again in the autumn, shortly after sowing of the winter crop.

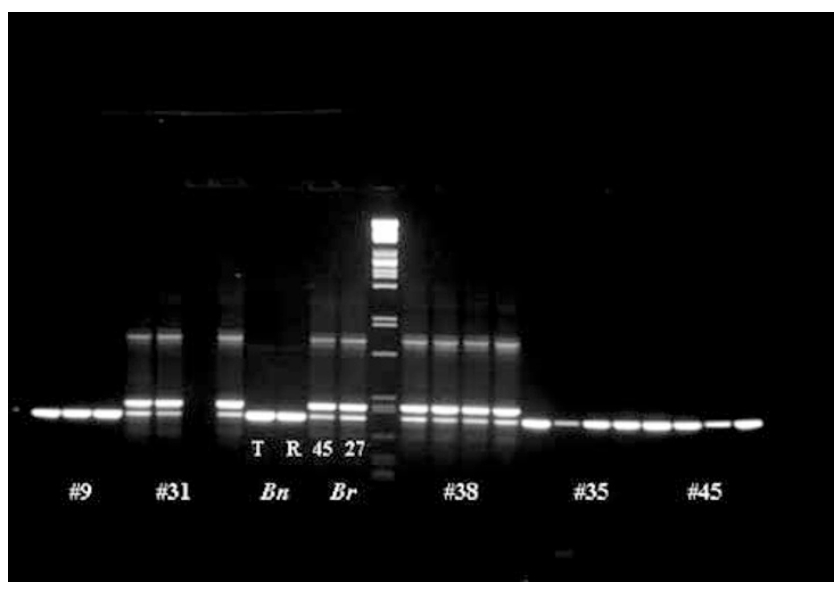

Figure 2 The species-specific cpDNA markers of B. napus, B. rapa and cpDNA typing of plants from the weedy population. 1-3: \#9 (oilseed rape according to AFLP profile); 4-5 and 7: \#31 (B. rapa according to AFLP profile); 8-9: B. napus (Topas and Rally); 10 and 11: B. rapa (Bc 45 and $\mathrm{Bc} 27$ ); 12: size marker; 13-16: \#38 (introgressed according to AFLP profile); 17-20: \#35 (B. rapa according to AFLP profile); 21-24: \#45 ( $\mathrm{F}_{1}$ hybrid according to AFLP profile).

Table 4 Species-specificity of the chloroplast marker in the mother plants from the natural population

\begin{tabular}{lrcc}
\hline & \multicolumn{3}{c}{ Assessment from AFLP-markers } \\
\cline { 2 - 4 } & B. r. & Introgressed & B. n. \\
\hline B. $r$. chloroplast & 47 & 34 & 0 \\
B. $n$. chloroplast & 1 & 2 & 7 \\
\hline
\end{tabular}

B.r.: Brassica rapa; B.n.: B. napus.
Concerning the chloroplast, only two of the 36 introgressed plants from the natural population had $B$. napus chloroplasts (Table 4 ), which suggests that $B$. rapa and introgressed plants with $B$. rapa-chloroplasts have functioned most often as the maternal plants in the introgression process. That hybridisation would be most successful when $B$. rapa is the maternal plant is also suggested by results from mixed pollinations (Hauser et al, 1997), since B. rapa pollen on B. napus styles had lower fitness than the conspecific pollen, whereas no difference between pollen types was found on $B$. rapa styles. Although B. napus presumably has a selection mechanism against $B$. rapa pollen, it seems that in the population studied here, the high pollen pressure from $B$. rapa and introgressed plants leads to the formation of $F_{1}$ hybrids or introgressed plants with B. napus as maternal parent (Table 3). On the other hand, the high preference of conspecific pollen in the $B$. napus plants, most likely hinders the elimination of this species. Therefore, there will still be B. napus plants present at low frequencies in the population, which ensures an ongoing production of hybrids and introgressed plants with the $B$. napus chloroplast and nuclear DNA.

Previous investigations have suggested that chloroplasts are maternally inherited in Brassica (Corriveau and Coleman, 1988; Scott and Wilkinson, 1999). The appearance of B. napus chloroplasts in one of the B. rapa-like plants (Table 4) is therefore likely to be the outcome of progressive introgression with $B$. napus as the maternal plant, at least once during the introgression process. As a minimum, two successive hybridisations are needed to produce a $B$. rapa-like plant with cpDNA from B. napus (Mikkelsen et al, 1996), suggesting that the introgression in the maternal population had been 
in progress for at least three plant generations (pure species, $\mathrm{F}_{1}$ and $\mathrm{BC}_{1}$ ). This is in accordance with previous results (Hansen et al, 2001). The finding of $B$. rapa-like plants with B. napus cytoplasm in population Bc 29 could be another example of this nuclear exchange process, where successive backcrosses with $B$. rapa ultimately produce plants that harbour a $B$. napus cytoplasm, two A genomes (part of these could be from $B$. napus) and no remains of the $\mathrm{C}$ genome (an interesting new origin of ' $B$. rapa'). Population Bc 29 was a very heavy infestation of $B$. rapa in oilseed rape. Also, at this site, the two species are known to have coexisted for years. In conclusion, these results imply a risk for transgene transfer from transplastomic lines of $B$. napus to $B$. rapa, although the frequency of transfer will be reduced compared to transgenic lines with a nuclear transgene position.

Scott and Wilkinson (1999) concluded that: 'chloroplast exchange (between $B$. napus and $B$. rapa) would be extremely rare and scattered' primarily because of low incidence of sympatry and inevitable population declines for feral $B$. napus. However, they also infer that, if '...mixed stands persist for long periods of time and hybridise freely, for instance, even a low rate of formation would be of significance'. In the present paper, a mixed population of $B$. napus and $B$. rapa was studied, which according to the owner of the organic field has existed for many years. This, and previous studies of the population (Landbo, 1997; Hansen et al, 2001), suggest that introgression has been in progress for at least three years, from 1995 to 1998. Sympatric populations are rather common in Danish fields. The oilseed rape plants in the organic field could be remains from conventional cultivation of the crop, and in contrast to feral oilseed rape populations they are maintained by the cultivation practice. Oilseed rape is probably also introduced as a weed in organic fields through sharing of combines, which is common practice for the smaller farms in Denmark. In organically cultivated fields, an agricultural annual has optimal conditions as there is no use of herbicides and ploughing will remove potential biannual or perennial competitors. From this study, it seems that when $B$. napus and $B$. rapa grow in mixed stands in a habitat with soil disturbance and poor weed control, there is a risk that introgression may lead to the production of $B$. rapa-like plants occasionally with an oilseed rape chloroplast.

In Denmark, B. rapa is a common weed of oilseed rape fields (Jørgensen and Andersen, 1994). The finding of chloroplast capture in a heavily infested field with conventional management (Bc 29, Figure 1) illustrates that introgression may also take place under traditional agricultural practice. In conclusion, these results suggest that the introgression process between $B$. napus and $B$. rapa primarily progressed with $B$. rapa as the maternal plant, and that both recombination between the $\mathrm{A}$ and $\mathrm{C}$ genomes and exchange of chloroplasts between $B$. napus and $B$. rapa appear to have occurred. The results suggest that when $B$. napus and $B$. rapa grow in mixed stands transgenes can be transferred from B. napus to B. rapa, regardless of transgene position in the nuclear or plastid DNA. In the UK, such mixed populations are apparently sporadic, ephemeral and likely to be widely dispersed (Scott and Wilkinson, 1999). However, in Denmark, B. rapa and the mixed populations with oilseed rape seem to be more common in agricultural settings.

\section{Acknowledgements}

The Danish Environmental Research Programme (SMPII, primary contributor), the Danish Research Agency (Centre for Bioethics and Risk Assessment) and The Nordic Joint Committee for Agricultural Research (NKJ) financed this project. We thank Bente Andersen and Rene Petersen for technical assistance.

\section{References}

Bateman AJ. (1955). Self-incompatibility systems in angiosperms. III Cruciferae. Heredity 9: 52-68.

Cheung WY, Champagne G, Hubert N, Landry BS (1997). Comparison of the genetic maps of Brassica napus and Brassica oleracea. Theor Appl Genet 94: 569-582.

Chévre AM, Eber F, Baranger A, Hureau G, Barret P, Renard M. (1998). Characterization of backcross generations obtained under field conditions from oilseed rape - wild radish $F_{1}$ interspecific hybrids: an assessment of transgene dispersal. Theor Appl Genet. 97: 90-98.

Corriveau JL, Coleman AW. (1988). Rapid screening method to detect potential biparental inheritance of plastid DNA and results for over 200 angiosperm species. Am J Bot 75: 1443-1458.

Daniell H, Datta R, Varma S, Gray S, Lee SB. (1998). Containment of herbicide resistance through genetic engineering of the chloroplast genome. Nat Biotechnol 16: 345-348.

Edwards K, Johnstone C, Thompson C (1991). A simple and rapid method for the preparation of plant genomic DNA for PCR analysis. Nucleic Acids Res 19: 1349.

Hansen LB, Siegismund HR, Jørgensen RB. (2001). Introgression between oilseed rape (Brassica napus L.) and its weedy relative B. rapa L. in a natural population. Genet Resources Crop Evol 48: 621-627.

Hauser TP, Jørgensen RB, Østergård H (1997). Preferential exclusion of hybrids in mixed pollinations between oilseed rape (Brassica napus) and weedy B. campestris (Brassicaceae). Am J Bot 84: 756-762.

Johannessen MM Mikkelsen TN, Jørgensen RB (2002). $\mathrm{CO}_{2}$ exploitation and genetic diversity in winter varieties of oilseed rape (Brassica napus); varieties of tomorrow. Euphytica 128: $75-86$.

Jørgensen RB Andersen B (1994). Spontaneous hybridization between oilseed rape (Brassica napus) and weedy B. campestris (Brassicaceae): a risk of growing genetically modified oilseed rape. Am J Bot 81: 1620-1626.

Lagercrantz U (1998). Comparative mapping between Arabidopsis thaliana and Brassica nigra indicates that Brassica genomes have evolved through extensive genome replication accompanied by chromosome fusions and frequent rearrangements. Genetics 150: 1217-1228.

Landbo L (1997). Introgression from Oilseed Rape (Brassica napus) to Weedy Brassica campestris - an Avenue for the Escape of Transgenes? PhD Thesis, Plant Biology and Biogeochemistry Department, Ris $\varnothing$ National Laboratory, Denmark.

Levin DA (1978). The origin of isolating mechanisms in flowering plants. Evol Biol 11: 185-317.

Linde-laursen I Bothmer R (1997). Effect of rye A and B chromosomes on meiotic association of Hordeum marinum ssp gussoneanum $(4 x)$ chromosomes in intergeneric hybrids. Hereditas 127: 193-201.

Maughan PJ, Saghai Maroof MA, Buss GR, Huestis GM (1996). Amplified fragment length polymorphism (AFLP) in soy- 
bean: species diversity, inheritance, and near-isogenic line analysis. Theor Appl Genet 93: 392-401.

Mikkelsen TR, Andersen B, Jørgensen RB (1996). The risk of crop transgene spread. Nature 380: 31 .

Olsson G (1952). Undersökning av graden av korsbefruktn-ing hos vitsenap och raps. Sveri Utsädesfören Tidsskrft 6: 311-322.

Scott SE, Wilkinson MJ (1999). Low probability of chloroplast movement from oilseed rape (Brassica napus) into wild Brassica rapa. Nat Biotechnol 17: 390-393.
Taberlet P, Gielly L, Pautou G, Bouvet J (1991). Universal primers for amplification of three non-coding regions of chloroplast DNA. Plant Mol Biol 17: 1105-1109.

U N (1935). Genomic analysis in Brassica with special reference to the experimental formation of B. napus and peculiar mode of fertilization. Jpn J Bot 7: 389-452.

Vos P, Hogers R, Bleeker M, Reijans M, Van De Lee T, Hornes M et al. (1995). AFLP: a new technique for DNA fingerprinting. Nucleic Acids Res 23: 4407-4414. 